\title{
Resource Allocation in a Generalized Framework for Virtualized Heterogeneous Wireless Network
}

\author{
Bo Fan, Hui Tian, Yuan Zhang, and Xiao Yan \\ State Key Laboratory of Networking and Switching Technology, Beijing University of Posts and Telecommunications, \\ Beijing 100876, China
}

Correspondence should be addressed to Hui Tian; tianhui@bupt.edu.cn

Received 9 December 2015; Revised 22 March 2016; Accepted 5 April 2016

Academic Editor: Lin Gao

Copyright (C) 2016 Bo Fan et al. This is an open access article distributed under the Creative Commons Attribution License, which permits unrestricted use, distribution, and reproduction in any medium, provided the original work is properly cited.

As a prevailing concept in $5 \mathrm{G}$, virtualization provides efficient coordination among multiple radio access technologies (RATs) and enables multiple service providers (SPs) to share different RATs' infrastructure. This paper proposes a generic framework for virtualizing heterogeneous wireless network with different RATs. A novel "VMAC" (virtualized medium access control) concept is introduced to converge different RAT protocols and perform inter-RAT resource allocation. To suit the proposed framework, a virtualization based resource allocation scheme is devised. We formulate the problem as a mixed combinatorial optimization, which jointly considers network access and rate allocation. First, to solve the network access problem, "adaptability ratio" is developed to model the fact that different RATs possess different adaptability to different services. And a Grey Relational Analysis (GRA) method is adopted to calculate the adaptability ratio. Second, services are modeled as players, bargaining for RAT resources in a Nash bargaining game. And a closed-form Nash bargaining solution (NBS) is derived. Combining adaptability ratio with NBS, a novel resource allocation algorithm is devised. Through simulation, the superiority and feasibility of the proposed algorithm are validated.

\section{Introduction}

Recent years are witnessing an unprecedented surge in mobile network services, causing a series of challenges to be confronted by mobile network operators (MNOs). MNOs have to increase network investments, including licensed spectrum, cell sites, and backhaul infrastructure to improve network capacity and fulfill users' needs. In addition, societal development and Internet of Things (IoT) will lead to many new application types, imposing increasingly diversified requirements to be serviced by MNOs in future mobile network system. To overcome these challenges, RAN (radio access network) virtualization is considered as a promising solution [1].

In RAN virtualization, service protocols are completely decoupled from the underlying RAN substrate: MNOs own and manage overall RAN resources, while service providers (SPs) compose network services by purchasing and sharing MNOs' RAN resources [2]. In order to achieve the RAN sharing, the 3GPP (Third Generation Partnership Project) releases two proposals [3]: spectrum-split sharing, where SPs share RANs according to different spectrum ranges; geography-split sharing, where SPs share RANs according to different geographical ranges. Another primary research is C-RAN (Cloud-RAN) [4]. In C-RAN, baseband signals are centrally processed in a cloud-based center unit (CU), with radio units (RUs) providing coverage to end users. Hence the RAN resources can be centrally processed and dynamically shared by different SPs. Although the flexibility of signal processing and resource utilization are theoretically maximized, fronthaul (the link between RU and CU) capacity limitation is known to impose a formidable bottleneck to C-RAN. Besides, several researches have set up testbed and commercial demonstration of virtualization based RANs, including IEEE 802.11 [5, 6], LTE [7], and WiMAX [8].

Within this thesis, resource allocation is an important issue. In [9], a bankruptcy game based resource allocation approach is investigated. Through the game, MNOs can dynamically share the wireless resources in LTE networks and the resource usage is proved to increase. Kalil et al. propose an 
efficient low-complexity scheduler to virtualize the wireless resource blocks (RBs) and share them among users of different MNOs [10]. The scheduler aims at maximizing the throughput while maintaining access proportional fairness (APF) among users as well as MNOs. However, the above researches focus on homogeneous networks, and the heterogeneity of user services is also ignored. Considering the fact that heterogeneous services as well as heterogeneous RATs coexist in future mobile networks, a virtualization based wireless resource allocation algorithm is proposed [11]. The algorithm conducts compensative resource allocation, where resources in a centralized resource pool are continuously reallocated to users of different MNOs to satisfy their service requirements.

As brilliant as the above works are, there still exist several disadvantages: First, there lacks a comprehensive methodology for virtualizing heterogeneous wireless network. Since virtual networks are created by SPs with various service requirements, virtualization systems have to bear multiple virtual networks that hold heterogeneous QoS (Quality of Service) requirements, topology, security, and so forth. In addition, MNOs with different RATs should be aggregated in the same virtualization substrate. Second, the adaptability of different networks to different services is ignored. For a certain RAT, there are multiple factors that affect the access or allocation of the RAT resources to different user services. For example, video stream requires short delay while data traffic is more sensitive to packet loss. However, existing works [911] consider resource allocation simply from the perspective of bandwidth or rate.

Therefore, in this paper, we propose a generalized framework for virtualized heterogeneous network with the major component of inter-RAT resource allocation. The main contributions can be concluded as follows.

We construct a generalized framework for virtualized heterogeneous network using the "VMAC" (Virtualized MAC) layer concept. Below VMAC, MNOs operate different networks of different RATs with their own PHY and MAC functions. VMAC acts as a middle layer, through which resources of different MNOs are aggregated within a cloudbased platform. Above VMAC, SPs provide end-to-end user services by applying network resources from the cloud.

A novel resource allocation strategy is devised to suit the proposed framework. Considering the heterogeneity of different services, we model the resource allocation problem as a Nash bargaining game subject to different service requirements. The problem is formulated as a mixed combinatorial optimization, which jointly considers network access and rate allocation.

To solve the network access problem, "adaptability ratio" is developed to model the adaptability of different networks to different services. And a Grey Relational Analysis (GRA) approach is used to calculate the "adaptability ratios," considering the influence of multiple network parameters (capacity, jitter, packet loss, etc.) to reach a practical solution. To solve the rate allocation problem, we transform the problem into a convex optimization and derive a closed-form Nash bargaining solution (NBS). Combining adaptability ratio with NBS, we develop a novel resource allocation algorithm, where services iteratively access the most suitable networks and bargain for the network resources. The algorithm is terminated when the overall service requirements are satisfied or network resources are used up. Through simulation, the superiority and feasibility of the proposed algorithm are validated by comparing with existing schemes.

The paper is organized as follows. The proposed virtualization framework is presented in detail in Section 2. The resource allocation strategy is given in Section 3. And the simulations are in Sections 4 and 5. Section 6 concludes the paper.

\section{The Proposed Virtualization Framework}

In this section, we first give an overview of the VMAC concept. After that, the proposed virtualization framework is presented in detail.

2.1. Why the Proposed VMAC? In existing approaches, when UE (user equipment) initiates handover or offloading over multiple RATs, multiple IP addresses will be assigned to one piece of UE to identify different RAT links. Each RAT link has a unique IP address without considering that these addresses belong to the same UE. These IP packets will be routed separately in the core network $(\mathrm{CN})$, generating different routing delays and causing disorders when multiplexing/demultiplexing them in low-layer RAN.

As a solution, VMAC is introduced as a converged network layer, which is tightly coupled into existing RAT protocol stacks with minimum protocol modifications. Through VMAC, heterogeneous RATs can be coupled within a unified protocol stack, forming the functionality for SPs to manage and operate their own virtualized networks.

Our aim is to provide seamless QoS and flexible resource usage. Through VMAC, offloading or handover protocols have maintained the RAT link processing at the VMAC layer, and UE IP is not changed when offloading or handover occurs. Thus, a stable seamless QoS classification of ongoing sessions can be guaranteed. And the different RAT resources can be flexibly aggregated or disassembled according to user service to realize the true sense of "network as a service."

2.2. A Converged Protocol Stack for Virtualized Heterogeneous Network. The proposed model is in Figure 1. VMAC is adopted to realize the converged protocol stack, which can merge and manage the different IP flows in a converged manner. The underlying MNOs provide different RATs with independent PHY and MAC functions, including modulation/demodulation, coding, measurement, and access. MNOs are linked through the management interface to a cloud-based platform, where resources from different MNOs can be abstracted out as a virtual RAN pool (VRP). VMAC is held within the cloud that is responsible for merging the packets from different RATs into a unified IP flow. The cloud is equipped with isolated memory to carry out its own computation tasks. SPs provide end-to-end user services on top of VMAC.

VMAC resides right above the network MAC layer, but below IP layer. The reasons are as follows. (1) In lower layer 


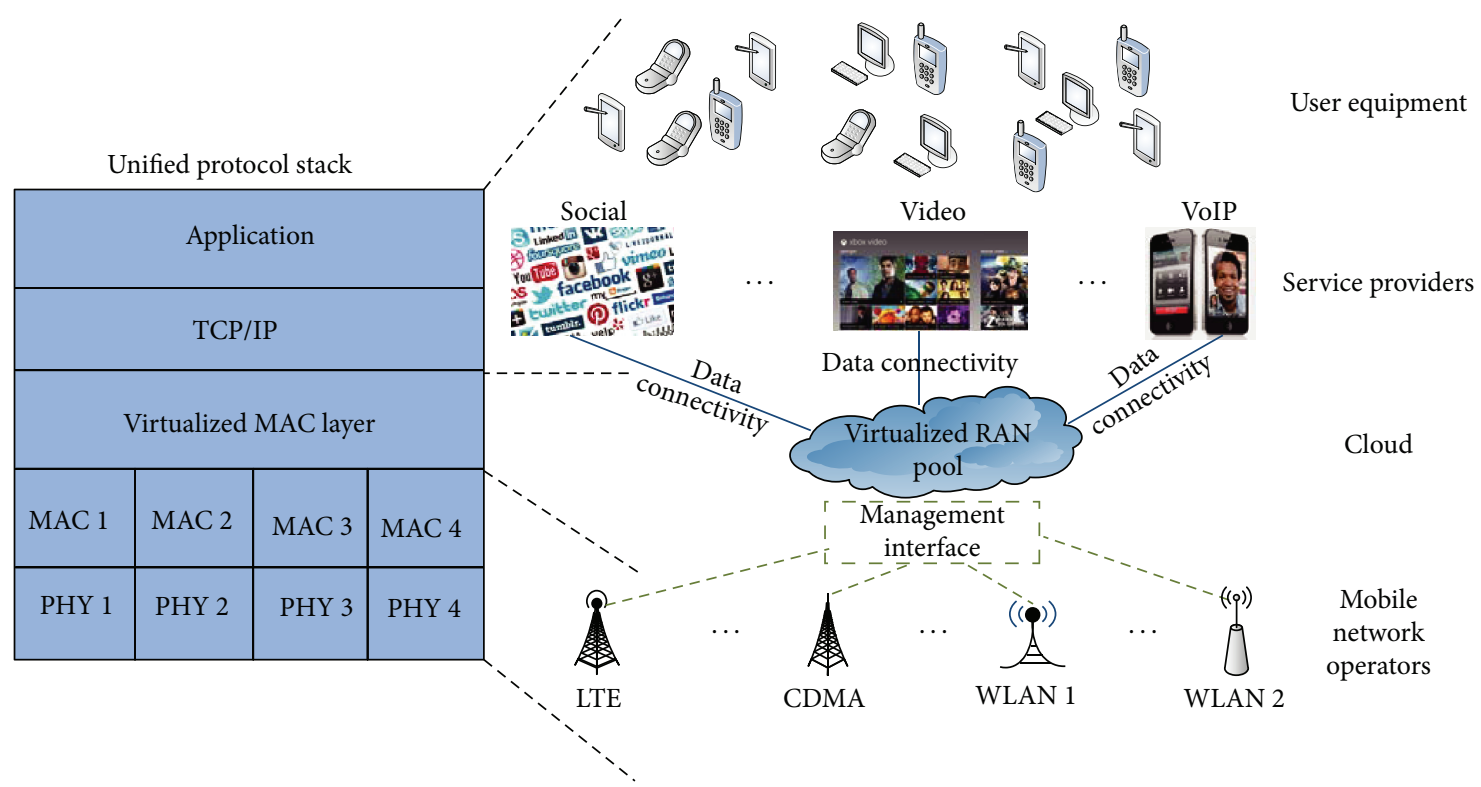

FIGURE 1: A converged protocol stack for virtualized heterogeneous network.

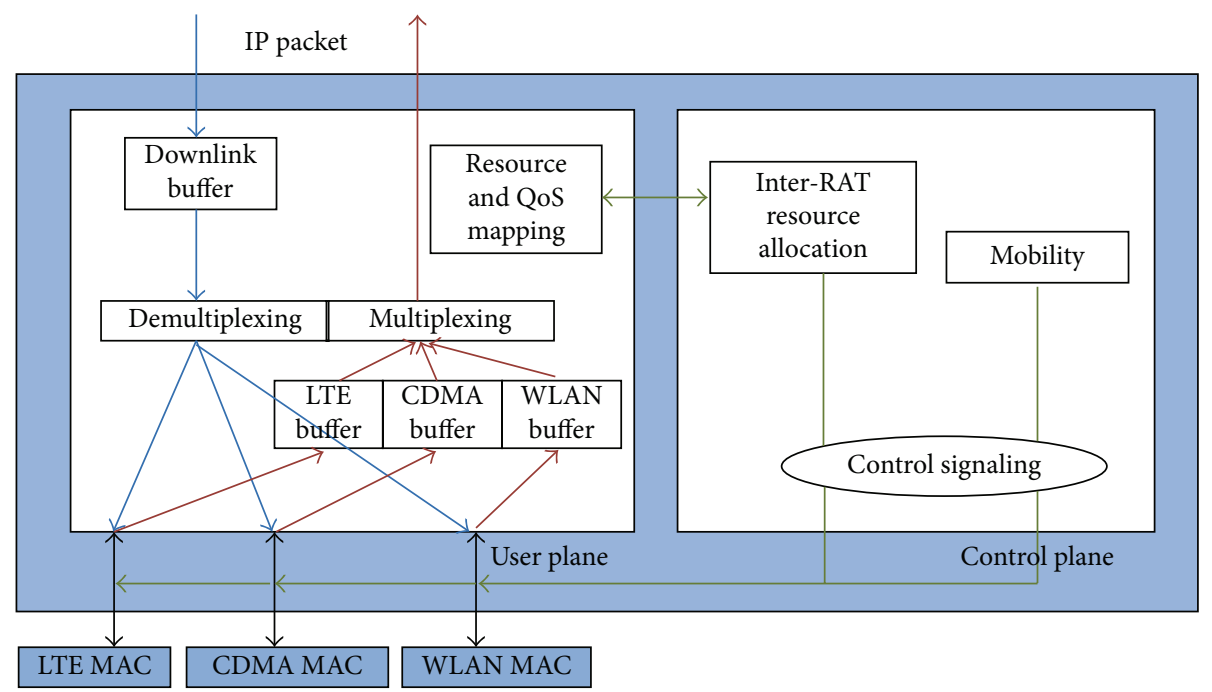

FIGURE 2: Functionalities of the Virtualized MAC layer.

virtualization (e.g., RF, PHY, and MAC layer), the functions of upper layers will be processed in a more centralized method. For example, in C-RAN, functions above RF, including signal processing, MAC QoS, and RRM management, are centralized [12]. Hence, the flexibility brought about by the centralized processing is maximized. However, there are two main drawbacks in low-layer virtualization. First, the backhaul overhead is tremendous and unbearable. Second, the QoS requirements of different RATs cannot be handled by a unified set of MAC control functionalities. (2) Higher layer virtualization (e.g., above IP) sustains the independence of different RATs, allowing multiple RATs to coexist within the same framework (compatibility). In conclusion, low-layer means more flexibility but less compatibility, and high-layer means less flexibility but more compatibility. As a tradeoff, the VMAC is proposed.

2.3. Functionalities of VMAC. The VMAC design consists in the functionalities of user and control planes, as shown in Figure 2.

2.3.1. User Plane. The VMAC user plane is responsible for demultiplexing the packets from $\mathrm{CN}$ into various RATs and merging the uplink packets from different RATs into a unified IP flow. In uplink, the UE protocol decides the bearing RAT of each IP packet and transmits the data to the VMAC through the respective RAT link. The VMAC encapsulates the data 
from different RATs and multiplexes several data flows into one common IP flow. In downlink, VMAC demultiplexes the service traffic to be carried by different RATs according to a predefined contract of the control plane. This contract defines for each piece of UE the relationship that exists between the business type of IP packet and the corresponding RAT and allocates packets to the corresponding RATs.

2.3.2. Control Plane. The right part of Figure 2 exemplifies VMC control plane, which defines RAT resource allocation and mobility management. Details are described as follows.

(i) QoS Mapping. As the range of services from SPs can be wide from bandwidth-consuming to delay-sensitive, the QoS of these different services must be dynamically mapped to the physical wireless links. Since different RATs have different capability and suitability in terms of different service types, such mappings need to provide seamless and suitable service quality across multiple RATs for user sessions.

(ii) Inter-RAT Resource Allocation. This module allocates RAT resources to SPs to support on-demand user services. The service packets can be accommodated simultaneously across multiple RATs or by a single RAT link. The proportion of packets between these RATs is determined on the basis of network and traffic characteristics. In our framework, multiple virtual networks (or SPs) coexist on the same network substrate. And SP may span over multiple underlying RATs to customize its unique business and service. Therefore, inter-RAT resource allocation module is responsible for elaborating specific solutions and mechanisms to support elastic provision, operation, and maintenance of the virtualized wireless networks.

(iii) Mobility Management. To maintain service continuity when a user switches among multiple RATs, proper mobility management mechanisms should be supported by the VMAC. There are two basic aspects in the mobility issue: location management and handoff.

\section{Resource Allocation Strategy}

In this part, we devise an inter-RAT resource allocation strategy for the proposed VAMC. In our model, the cloud acts on behalf of the resources in the VRP and decides the utilization of these resources in order to satisfy SPs' service requests. Since there exist multiple services and RAT types and different RATs can have different adaptability to different services, it is important to consider this "adaptability" as a benchmark for designing the resource allocation strategy. Therefore, in this section, we devise an "adaptability ratio" concept to solve the resource allocation problem. The problem is formulated using Nash bargaining game, and we develop a two-step solution. In Step 1, different services select their most suitable RATs with the highest adaptability ratios. In Step 2, services are modeled as players, bargaining for the resources of the selected RATs in Step 1. Based on the twostep solutions, an iterative resource allocation algorithm is devised.

3.1. Problem Formulation. Our focus is to identify the SPs' service requests and accordingly allocate MNOs' RAT resources. And we formulate the resource allocation using Nash bargaining game. Nash bargaining is chosen considering the following reasons: First, in our model, there are multiple services with different characteristics. Nash bargaining provides an efficient resource allocation framework to the different services taking into account their different needs and performance requirements. Second, Nash bargaining provides an efficient solution considering the allocation fairness. In addition, the complexity of the Nash bargaining solution (NBS) is comparatively low.

Nash bargaining is a type of negotiation in which players compete for limited resources and try to reach an agreement that has mutual benefits among them [13]. If the total resources requested by players are less than the available ones, all the players' requests are satisfied. Otherwise, the negotiation is compromised and all players will only obtain the guaranteed resource determined by the bargaining game. In our model, there are I services bargaining for RAN capacities, each with a minimum rate $\left(\mathrm{MR}_{i}\right)$ and a peak rate $\left(\mathrm{PR}_{i}\right)$ constraint. RANs are indexed by $j \in\{1, \ldots, J\}$ with capacity $C_{j}$.

Normally, the solution of a Nash bargaining game is given by NBS. For all $i, j$, let $\mathbf{u}=\left(u_{11}, u_{12}, u_{i j}, \ldots, u_{I J}\right)$ represent resource allocation vector. $\mathcal{U} \subset \mathscr{R}^{I \times J}$ denote the set of achievable rate, which is a nonempty closed upper-bounded real-number space (where $\mathscr{R}^{I \times J}$ denote $I \times J$ dimensioned real-number field). The notion of NBS is given as follows [13].

Definition 1 (Nash bargaining solution). Let $S$ be a mapping defined as $S:\left(\mathcal{U}, \mathrm{MR}_{i}\right) \rightarrow \mathscr{R}^{I \times J}$. The NBS is a unique bargaining solution $\mathbf{u}^{*}=S\left(\mathcal{U}, \mathrm{MR}_{i}\right)$ if the following conditions are satisfied:

(1) $\mathbf{u}^{*} \in \mathcal{U}$.

(2) $\mathbf{u}^{*}$ is Pareto optimal.

(3) Independence of linear transformations: if $\varphi$ is a given linear mapping, then $S\left(\varphi(\mathcal{U}), \varphi\left(\mathrm{MR}_{i}\right)\right)=$ $\varphi\left(S\left(\mathcal{U}, \mathrm{MR}_{i}\right)\right)$.

(4) Independence of irrelevant alternatives: if $\mathscr{V} \subset \mathcal{U}$ and $S\left(\mathcal{U}, \mathrm{MR}_{i}\right) \in \mathcal{U}$, then $S\left(\mathcal{U}, \mathrm{MR}_{i}\right)=S\left(\mathscr{V}, \mathrm{MR}_{i}\right)$.

(5) There is symmetry.

Conditions (1) and (2) guarantee the existence and efficiency of the NBS. The implication of a Pareto optimum is that it is impossible to find another point which leads to strictly superior performance for all the service allocation simultaneously. Conditions (3)-(5) guarantee NBS fairness. Condition (3) implies that the bargaining solution is scale invariant; that is, the bargaining solution is unchanged if the performance objectives are linearly scaled. Condition (4) states that the bargaining point is not affected by enlarging the domain if agreement can be found on a restricted domain. 
Symmetry condition (5) guarantees equal priority for the players with the same initial points and objectives. If the symmetry axiom is satisfied, then all the players involved in the bargaining game will be assigned the same amount of resources. However, in our model, since the budget represents service rate requirement, it may not be reasonable when the budgets of players are significantly different. So the generalized NBS is a variant of the NBS by assigning players with different budgets, which is defined in [14] as follows.

Definition 2. Let $B_{i}$ denote the budget of player $i$. Then the optimal and fair NBS is given by the unique solution to

$$
\max _{\mathbf{a}, \mathbf{u}} \prod_{i=1}^{I}\left(\sum_{j=1}^{J} a_{i j} u_{i j}-\mathrm{MR}_{i}\right)^{B_{i}} .
$$

In our model, each service should be guaranteed a minimum rate $\left(\mathrm{MR}_{i}\right)$ and a peak rate $\left(\mathrm{PR}_{i}\right)$. The minimum rate represents the minimum resource to maintain the service provision and could be tariffs published by the cloud (e.g., gold, silver, or bronze services, which can guarantee different levels of service priority [15]). Resource allocation index $a_{i j}$ is equal to 1 if service $i$ has access to RAN $j$ and 0 otherwise. a denote the vector of $a_{i j}$.

In conventional NBS, the resource allocation is simply considered from the perspective of bandwidth or transmission rate. However, we argue that bandwidth and other network parameters like latency, packet loss, and jitter are all important to QoS. In addition, the derivation of NBS is based on the assumption of given a. But in our model, it is important to identify the allocation or access priority of different services to different RATs, considering their different characteristics and requirements.

Therefore, we want to solve the problem in (1) from a more comprehensive perspective and improve conventional NBS. So we reformulate our resource allocation problem as

$$
\begin{array}{cc}
\max _{\mathbf{a}, \mathbf{u}} & \prod_{i=1}^{I}\left(\sum_{j=1}^{J} a_{i j} u_{i j}-\mathrm{MR}_{i}\right)^{B_{i}} \\
\text { s.t. } \quad \mathrm{C} 1: \sum_{j=1}^{J} a_{i j} u_{i j} \geq \mathrm{MR}_{i}, \quad \forall i \\
\mathrm{C} 2: \sum_{j=1}^{J} a_{i j} u_{i j} \leq \mathrm{PR}_{i}, \quad \forall i \\
\mathrm{C} 3: \sum_{i=1}^{I} a_{i j} u_{i j} \leq C_{j}, \quad \forall j \\
\text { C4: } a_{i j} \in\{0,1\}, \quad \forall i, j,
\end{array}
$$

where a with element $a_{i j}$ and $\mathbf{u}$ with element $u_{i j}$ are the network access index and rate allocation strategy. $\mathrm{C} 1$ and $\mathrm{C} 2$ are the service rate constraints and C3 denotes the network capacity constraint. The allocation problem is a mixed combinatorial optimization problem, which is complex to solve. For simplicity, we develop an iterative algorithm to solve it.
Each iteration consists of the two substeps: First, users of different services access their most suitable networks based on the service requirements. Second, after users access, the rate allocation of different networks to different user services is determined.

3.2. Step 1: "Adaptability Ratio" Based Network Access. In Step 1, we want to determine the network access of different user services to different RATs. Since different networks have different performances in terms of latency, bandwidth, rate, and so forth, different services will have different access priorities for different networks considering these performance factors. Hence, we introduce an "adaptability ratio" concept to measure the adaptability of different services to different networks.

Since there are multiple performance factors to be considered in a certain network, we want to give a comprehensive evaluation on these factors to obtain an efficient access strategy. Therefore, we adopt GRA method to calculate the adaptability ratios. GRA is proved to be an efficient methodology for multicriteria evaluation [16]. GRA can make a fast decision with comparatively low complexity when there are multiple factors to be considered. In addition, GRA can minimize the influence of subjective factors and make a comprehensive decision through comparing and evaluating the multiple factors.

Adaptability ratio $\eta_{i j}$, which shows the adaptability of $j_{\text {th }}$ RAN to $i_{\text {th }}$ service, is defined as a real number in the range of $[0,1]$. The greater $\eta_{i j}$ is, the more suitable the network is for the transmission of the service traffic. And the calculations can be performed in the following 5 steps [16]:

(1) Classify the networks parameters by two situations (the-smaller-the-better, the-larger-the-better).

(2) Define the upper and lower bounds of the parameters.

(3) Normalize the parameters.

(4) Calculate the GRC (Grey Relational Coefficient).

(5) Normalize the GRC and obtain the adaptability ratio.

The RAN parameters we consider include the-smallerthe-better (latency, jitter, etc.) and the-larger-the-better (bandwidth, capacity, etc.) parameters. As for each RAN $j$, there are $K$ performance parameters and $w_{j, k}$ represents the performance value of the $k_{\text {th }}$ parameter for the $j_{\text {th }}$ RAN. We define $U_{k}$ and $L_{k}$ as the upper and lower bound for the $k_{\text {th }}$ parameter, respectively. The normalization formulas for the-smaller-the-better parameters and the-larger-the-better parameters are $w_{j, k}{ }^{*}=\left(U_{k}-w_{j, k}\right) /\left(U_{k}-L_{k}\right)$ and $w_{j, k}{ }^{*}=$ $\left(w_{j, k}-L_{k}\right) /\left(U_{k}-L_{k}\right)$, respectively.

For every RAN $j$, the normalized parameters can be written in a vector $w_{j}{ }^{*}=\left[w_{j, 1}{ }^{*}, w_{j, 2}{ }^{*}, \ldots, w_{j, K}{ }^{*}\right]$. And the GRC can be obtained as [16]

$$
\mathrm{GRC}_{i j}=\frac{1}{\sum_{k=1}^{K} \varepsilon_{i, k}\left|w_{j, k}^{*}-1\right|+1}
$$

Here $\varepsilon_{i, k}$ represents the preference coefficient of the $i_{\text {th }}$ service to the $k_{\text {th }}$ parameter, which can be determined by 
the characteristics of the service type. The more the emphasis the $i_{\text {th }}$ service put on the $k_{\text {th }}$ parameter is, the larger $\varepsilon_{i, k}$ will be [16]. We can put $\mathrm{GRC}_{i j}$ in a matrix, which has $I$ rows and $J$ lines. It is obvious that $\mathrm{GRC}_{i j} \in(0,1]$ and the most approximate RAN for $i_{\text {th }}$ service has the biggest $\mathrm{GRC}_{i j}$ value in the $i$ th row. As for each service $i$, the adaptability ratio can be obtained via normalizing the $\mathrm{GRC}_{i j}$ by the biggest value in each row

$$
\eta_{i j}=\frac{\mathrm{GRC}_{i j}}{\max _{j} \mathrm{GRC}_{i j}}
$$

Using (4), we can solve the network access index, which is shown in Algorithm 1, lines (3)-(8). The idea is very straightforward: each service tends to obtain resources from the network with highest $\eta_{i j}$ from the available resources in the VRP. Hence, the network with the best adaptability ratio is selected for each service.

3.3. Step 2: Rate Allocation. Given $a_{i j}$, we can transform the original problem into an equivalent concave optimization problem and obtain the optimal rate allocation $u_{i j}$ by the Lagrange dual approach. Based on log-function properties, the objectives in (2) can be transformed to log-form with the problem formulated as

$$
\begin{array}{ll}
\max _{\mathbf{u}} & \sum_{i=1}^{I} B_{i} \ln \left(u_{i j}-\mathrm{MR}_{i}\right) \\
\text { s.t. } & \mathrm{MR}_{i} \leq u_{i j} \leq \mathrm{PR}_{i}, \quad i \in\{1,2, \ldots, I\} \\
& \sum_{i=1}^{I} a_{i j} u_{i j} \leq C_{j}, \quad j \in\{1,2, \ldots, J\} .
\end{array}
$$

Since the transformed problem is convex, the optimal solution can be obtained by solving the Karush-Kuhn-Tucker (KKT) conditions. By introducing $\lambda_{i}, v_{i}$, and $\tau_{j}$ as Lagrange multipliers, we can obtain the following Lagrangian function:

$$
\begin{aligned}
L\left(u_{i j}, \lambda_{i}, v_{i}, \tau_{j}\right)= & \sum_{i=1}^{I} B_{i} \ln \left(u_{i j}-\mathrm{MR}_{i}\right) \\
& -\sum_{i=1}^{I} \lambda_{i}\left(\mathrm{MR}_{i}-u_{i j}\right) \\
& -\sum_{i=1}^{I} v_{i}\left(u_{i j}-\mathrm{PR}_{i}\right) \\
& -\sum_{j=1}^{J} \tau_{j}\left(\sum_{i=1}^{I} a_{i j} u_{i j}-C_{j}\right) .
\end{aligned}
$$

And the KKT conditions of (5) can be therefore written as

$$
\begin{aligned}
\frac{\partial L}{\partial u_{i j}} & =0, \quad \forall i \in\{1,2, \ldots, I\}, \\
\lambda_{i}\left(\mathrm{MR}_{i}-u_{i j}\right) & =0, \quad \forall i \in\{1,2, \ldots, I\}, \\
v_{i}\left(u_{i}-\mathrm{PR}_{i}\right) & =0, \quad \forall i \in\{1,2, \ldots, I\}, \\
\tau_{j}\left(\sum_{i=1}^{I} a_{i j} u_{i j}-C_{j}\right) & =0, \quad \forall j \in\{1,2, \ldots, J\} .
\end{aligned}
$$

Under the assumption $\sum_{i=1}^{I} a_{i j} \mathrm{MR}_{i} \leq C_{j}$, we observe that constraints $u_{i j}>\mathrm{MR}_{i}$ are nonactive and hence $\lambda_{i}=0$. Furthermore, $v_{i}=0$ if $u_{i j}<\mathrm{PR}_{i}$ and $u_{i j}=\mathrm{PR}_{i}$ otherwise. Based on the above analysis and KKT conditions in (7), we can derive that

$$
\begin{aligned}
\frac{\partial L}{\partial u_{i j}} & =\frac{B_{i}}{u_{i j}-\mathrm{MR}_{i}}+\lambda_{i}-v_{i}-\sum_{j=1}^{J} a_{i j} \tau_{j}=0 \Longrightarrow \\
u_{i j} & =\mathrm{MR}_{i}+\frac{B_{i}}{\sum_{j=1}^{J} a_{i j} \tau_{j}} .
\end{aligned}
$$

And then we have

$$
\sum_{i=1}^{I} a_{i j} u_{i j}-C_{j}=0, \quad \forall j \in\{1,2, \ldots, J\} .
$$

By substituting (8) into (9), we obtain

$$
\begin{aligned}
\sum_{i=1}^{I} a_{i j}\left(\mathrm{MR}_{i}+\frac{B_{i}}{\sum_{j=1}^{J} a_{i j} \tau_{j}}\right)-C_{j}=0, & \\
& \forall j \in\{1,2, \ldots, J\} .
\end{aligned}
$$

It can be observed that (10) is an equation set where $\tau_{j}$ can be uniquely determined for arbitrary $j$. Finally the optimal solution can be expressed as

$$
u_{i j}=\mathrm{MR}_{i}+\min \left(\mathrm{PR}_{i}-\mathrm{MR}_{i}, \frac{B_{i}}{\sum_{j=1}^{J} a_{i j} \tau_{j}}\right) .
$$

3.4. The Proposed Resource Allocation Algorithm. So far, we have solved the resource allocation problem in (1). However, we argue that the solutions obtained by the proposed steps are not optimal. This is because, in Step 1, each service is assumed to access only one network with the best adaptability ratio. In fact, the unsatisfied services will continue to access other networks even if they have comparatively lower adaptability ratios. Hence, we propose an iterative algorithm where the two-step bargain solutions are iteratively computed to obtain the optimal resource allocation results. The optimal results guarantee that one service can transmit through multiple RATs and one RAT can serve multiple services simultaneously. The algorithm pseudocode is given in Algorithm 1. 
(1) Initialize $u_{i j}, a_{i j}=0, \forall i \in\{1, \ldots, I\}, j \in\{1, \ldots, J\}$

(2) repeat

(3) Step 1:

(4) for $i=1: I$ do

(5) if $u_{i}<B_{i}$ then

(6) $\quad j=\left\{j \mid \max \left\{\eta_{i j}, \forall j\right\} \wedge C_{j} \neq 0 \wedge B_{j} \neq 0\right\}, a_{i j}=1$

(7) end if

(8) end for

(9) Step 2:

(10) Solve $u_{i j}$ as in (11)

(11) $\forall i, j$, update $B_{i}=\left[B_{i}-u_{i j}\right]^{+}$,

$\mathrm{MR}_{i}=\left[\mathrm{MR}_{i}-u_{i j}\right]^{+}, \mathrm{PR}_{i}=\left[\mathrm{PR}_{i}-u_{i j}\right]^{+}$,

$C_{j}=\left[C_{j}-\sum_{i=1}^{I} a_{i j} u_{i j}\right]^{+}, a_{i j}=0$

(12) until $\left(B_{i}=0, \forall i\right) \vee\left(C_{j}=0, \forall j\right)$

Algorithm 1: Iterative bargaining algorithm.

As for each iteration, the algorithm executes the proposed two steps. Step 1 corresponds to lines (4)-(8), where $a_{i j}$ is calculated based on the "adaptability ratios" $\eta_{i j}$. Step 2 corresponds to line (10), where the rate allocation is calculated based on the Nash bargaining solution. Finally, service satisfaction and resource usage should be updated, as in line (11). And this information will be utilized to generate the resource allocation results in the next iteration. This guarantees that, in the subsequent bargaining procedures, the services which have been allocated resources will lower their priorities, and occupied resources will be no longer available. The algorithm will be terminated when either the services are fully satisfied or the resources are completely used up. Note that $[x]^{+}$denotes $\max \{0, x\}$.

3.5. Complexity Analysis. The algorithm complexity depends on the total service number $I$ and network number $J$. Consider the worst case that the service rate requests are far greater than the network capacity, and the services have similar access priorities to different networks. Thus, in each iteration, the services will simultaneously access the same network. And the algorithm will be terminated when all networks are bargained for once. So the complexity of the algorithm is $O(I J)$.

\section{Simulation Configuration}

In the simulation, a square area $(100 \mathrm{~m} \times 100 \mathrm{~m})$ is cocovered by two LTE-macro-cells and two WLAN-APs (access points), that is, LTE-macro-cell 1, LTE-macro-cell 2, WLAN-AP 1, and WLAN-AP 2. The specific channel conditions (path loss, shadow, and fast-fading) for LTE and WLAN follow the standards in $[14,17] .60$ mobile users are randomly distributed within the area, each with a VoIP service. Among the total users, 40 users are with data service and 30 users are with video service. The three service types configured by the numeric pair (MR, PR, budget) in units of Mbps are as follows: $\operatorname{VoIP}(0.03,0.12,0.10)$, data $(0.10,0.70,0.50)$, and video $(0.30,1.50,1.20)$.
Two benchmarks are given for comparison. (1) The first one is "legacy" network setup without virtualization. In legacy network setup, MNOs operate isolated from each other and their resources cannot be shared. Service priority is VoIP $>$ video $>$ data and resource scheduler is proportionally fair. (2) The second one is CRA (compensative resource allocation) in [11], where resources from the VRP are continuously allocated to unsatisfied services. CRA is multiple-RAT and multiple-service resource allocation in virtualization based heterogeneous wireless network.

To evaluate the rate allocation fairness of the algorithm, Jain's fairness is introduced as $\psi=\left(\sum_{i=1}^{I} r_{i}\right)^{2} /\left(I \cdot \sum_{i=1}^{I} r_{i}^{2}\right)$ [18], where $r_{i}=\sum_{j=1}^{J} \eta_{i j} u_{i j} / B_{i}$ denotes service satisfaction in terms of the allocated rate relative to its budget. $\psi \in[0,1]$ and larger $\psi$ means services benefit from uniform satisfaction factor $r_{i}$.

To evaluate the resource utilization of the algorithm, resource utilization index is introduced. As for each RAN $j$, its resource utilization index is defined as $\sigma_{j}=\sum_{i=1}^{I} \eta_{i j} u_{i j} / C_{j}$. And the average utilization of the $J$ RANs can be calculated as $\sigma=\sigma_{j} \sum_{j=1}^{J} \sigma_{j} / J$.

\section{Performance Analysis}

5.1. Resource Allocation Results. We conduct 1000 simulation periods (transmission periods). And Table 1 gives a snapshot of the network parameters in a certain transmission period (of the total 1000 transmission periods). Capacity, latency, packet loss, and jitter are dynamic parameters. They can be calculated based on the resource allocation and service traffic condition of the current simulation period (assuming period $n$ ). Hence the calculated adaptability ratios are also dynamic. Table 2 gives a snapshot of the calculated adaptability ratios (in line with Table 1). These adaptability ratios are utilized to generate the resource allocation in the next simulation period (period $n+1)$.

Figure 3 gives the proposed resource allocation results in simulation period $n+1$. The allocation is determined based on the calculated adaptability ratios (as given in Table 2) in simulation period $n$. It can be observed that LTE-macro-cell is 


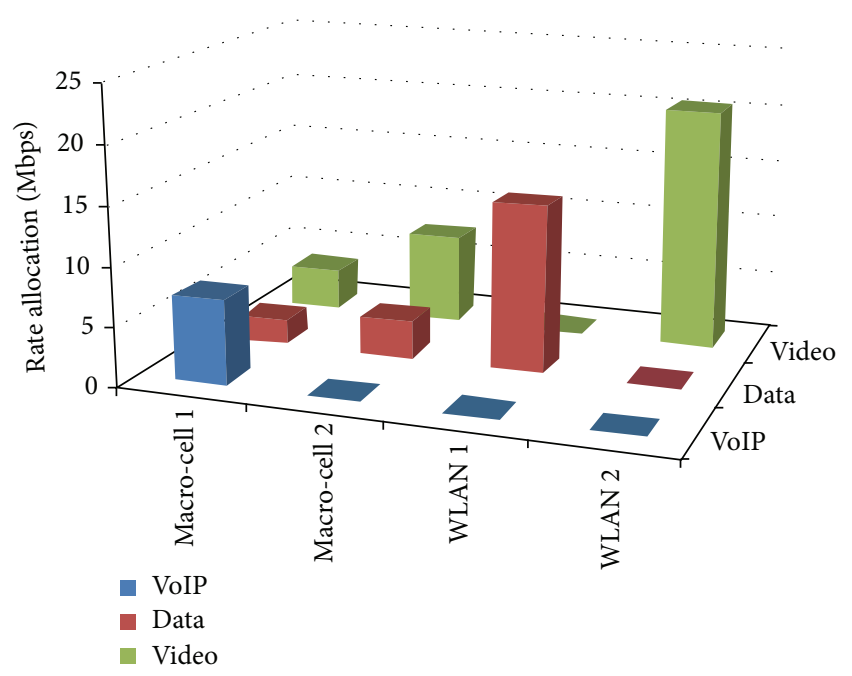

Figure 3: A snapshot of resource allocation results.

TABLE 1: A snapshot of network parameters.

\begin{tabular}{lcccc}
\hline & Capacity & Latency & Packet loss & Jitter \\
\hline LTE-macro-cell 1 & $12.7 \mathrm{Mbps}$ & $25 \mathrm{~ms}$ & $3 \%$ & $4 \mathrm{~ms}$ \\
LTE-macro-cell 2 & $10.8 \mathrm{Mbps}$ & $65 \mathrm{~ms}$ & $1 \%$ & $3 \mathrm{~ms}$ \\
WLAN-AP 1 & $14.1 \mathrm{Mbps}$ & $100 \mathrm{~ms}$ & $1 \%$ & $3 \mathrm{~ms}$ \\
WLAN-AP 2 & $20.5 \mathrm{Mbps}$ & $145 \mathrm{~ms}$ & $2 \%$ & $2 \mathrm{~ms}$ \\
\hline
\end{tabular}

TABLE 2: A snapshot of calculated adaptability ratios.

\begin{tabular}{lcccc}
\hline & Macro-cell 1 & Macro-cell 2 & WLAN 1 & WLAN 2 \\
\hline VoIP & 1.00 & 0.93 & 0.85 & 0.74 \\
Data & 0.91 & 0.95 & 1.00 & 0.98 \\
Video & 0.76 & 0.78 & 0.85 & 1.00 \\
\hline
\end{tabular}

best for VoIP, and VoIP is totally serviced by LTE-macro-cell, as in Figure 3. Besides, services tend to obtain more resource chunks from RANs with better adaptability ratio.

To better understand the resource allocation algorithm, we explain the detailed resource allocation procedure in simulation period $n+1$ as follows (i.e., how the results in Figure 3 are obtained). The allocation results in Figure 3 are obtained through 3 total iterations under the proposed algorithm. In the 1st iteration, according to the calculated "adaptability ratios" in Table 2, services access the most suitable networks and bargain for resources according to the derived solution in (11). Hence macro-cell 1 completely serves VoIP, WLAN 1 completely serves data, and WLAN 2 completely serves video. Since overall VoIP rate requirements are less than the capacity of macro-cell 1 , VoIP services are totally satisfied and will not enter the followed iterations. Since WLAN 2 capacity is less than the overall video rate requirements, WLAN 2 is totally subscribed to video services and will be unavailable in the followed iterations. The relation between WLAN 1 and data is the same as WLAN 2 and video.

In the 2nd iteration, the unsatisfied services continue to access the available networks even with the suboptimal adaptability. Hence, data and video simultaneously access

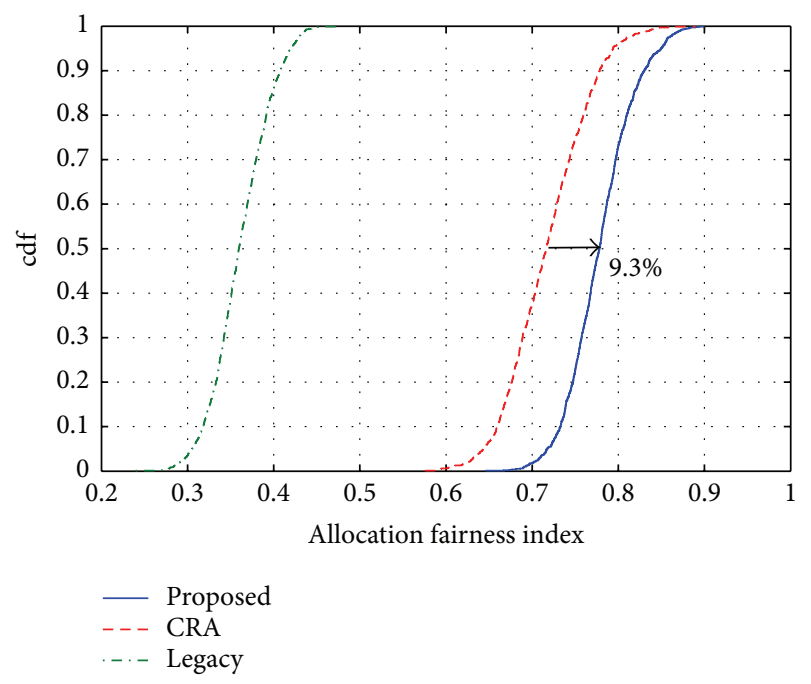

FIGURE 4: Comparison of cdf curves of the fairness index under different resource allocation schemes.

macro-cell 2 and bargain for network resources. After that, resources of macro-cell 2 are used up while the data and video requirements are still unsatisfied. Similarly, in the 3rd iteration, data and video access and bargain for resources of macro-cell 1. After that, the algorithm is terminated because the overall network resources are used up. Through iterations $1-3$, we can obtain the overall results of the resource allocation, as presented in Figure 3.

5.2. Performance Comparison. Figure 4 compares the cdf (cumulative distribution function) of the allocation fairness index under different resource allocation schemes. Virtualization based schemes (the proposed scheme and CRA) significantly outperform the legacy network. This is because, in legacy network setup, resources of each MNO can only be allocated to its subscribed users. Hence redundant resources will be wasted, deteriorating network efficiency. In addition, compared with CRA, the average resource utilization improves $9.3 \%$ through the proposed resource allocation algorithm.

Figure 5 compares the cdf of the resource utilization through different resource allocation schemes. The proposed scheme significantly outperforms CRA and legacy network. This is because "adaptability ratios" are used to determine efficient resource allocation, considering the influence of multiple network parameters. In comparison, CRA only considering network capacity is not efficient enough. Unsatisfied services cannot use the abundant resources owned by other MNOs.

Furthermore, to verify the feasibility of the proposed algorithm, we conduct the simulation by changing the rate requirement of video service.

5.3. Dynamic Video Service. Figure 6 demonstrates the rate allocation of different services as video service requirement varies. Before point 1 , the network capacity is adequate to meet services' requirement. Afterwards, the network 


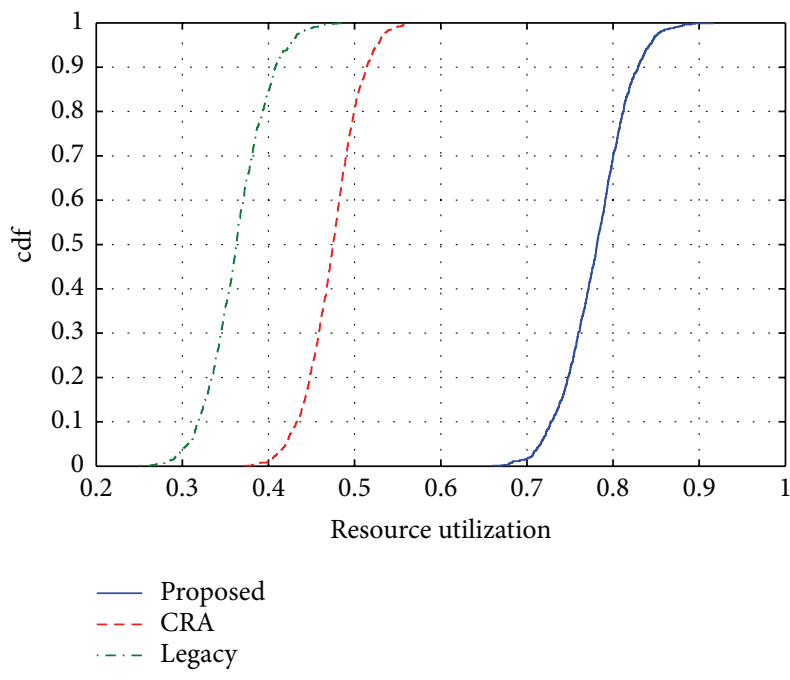

FIGURE 5: Comparison of cdf curves of the resource utilization under different resource allocation schemes.

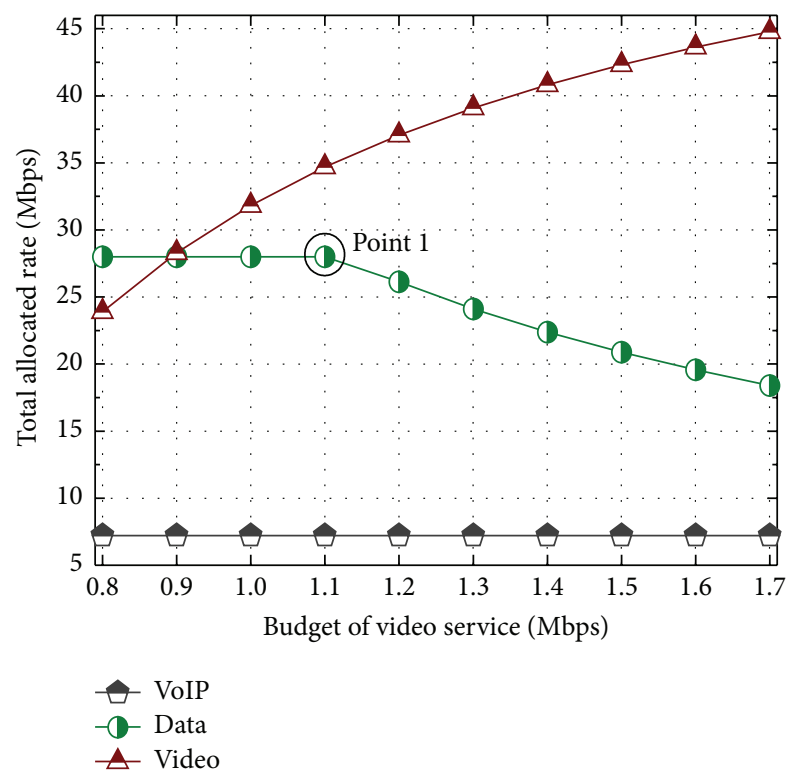

FIGURE 6: Service rate allocation of the proposed algorithm as the budget of video service varies.

becomes congested and the capacity allocation is performed in relation to different services' budgets. It should be noted that rate allocation of VoIP remains steady due to two reasons: First, overall VoIP's rate requirements are less than the LTE network capacity. Second, compared with data and video, VoIP has higher priority. Therefore, VoIP services can be totally satisfied by using the LTE-macro-cell resources.

Figure 7 shows the fairness index under different allocation schemes as video service requirement varies. As requirement of video service increases, the gap between the total service requirement and network capacity increases. Hence the fairness index decreases. The proposed scheme achieves the best fairness index by taking advantage of

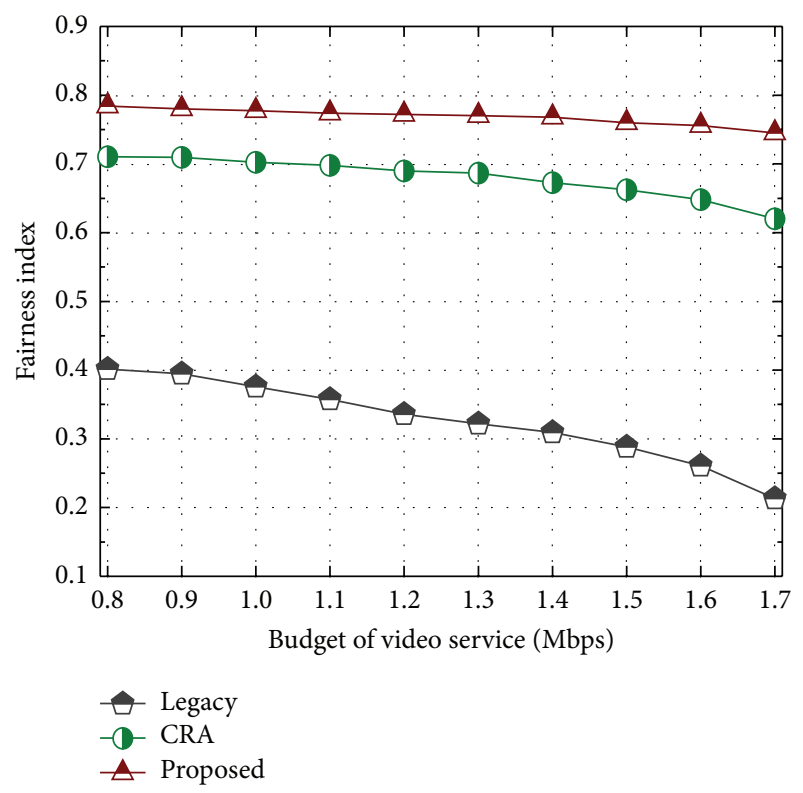

FIgURE 7: Comparison of fairness indexes as the budget of video service varies.

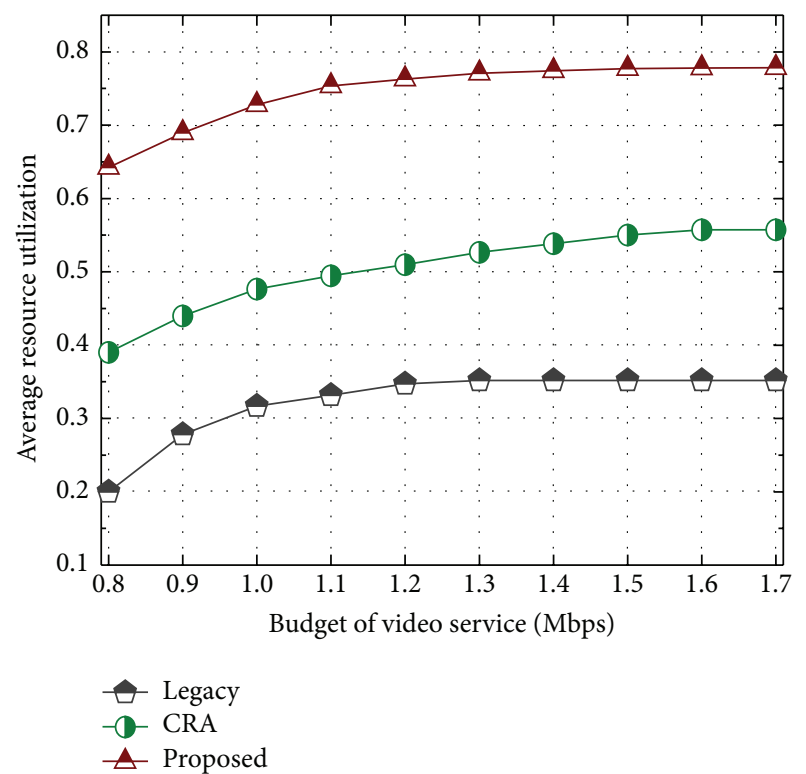

FIGURE 8: Evaluation of resource utilization as the budget of video service varies.

the centralized RAT processing and optimized allocation according to different network-service adaptability ratios.

Figure 8 shows the resource utilization under different allocation schemes as video service requirement varies. At first, the total network capacities are greater than total service requirements, which leaves redundant network capacities unoccupied. As the service requirement increases, the unoccupied capacities are utilized, and therefore the network resource utilization is increased. Note that the legacy scheme converges faster to stability because the video service is subscribed to one single network. Capacities from the other 
network(s) cannot be utilized even when the resources of the subscribed network are used up, hence limiting the resource utilization.

\section{Conclusions}

Wireless network virtualization is promising to solve the ossification of today's mobile network setup. This paper proposes a generalized virtualization framework for heterogeneous wireless network. The "VMAC" concept and VMAC data plane and control plane functions are devised to achieve virtualization over heterogeneous wireless networks. Furthermore, a resource allocation strategy is devised to suit the virtualization framework. "Adaptability ratios" are introduced to model network suitability to different services. Combining "adaptability ratios" with Nash bargaining, an iterative resource allocation algorithm is devised. Through simulation, the advantages of the proposed algorithm are validated.

\section{Competing Interests}

The authors declare that they have no competing interests.

\section{Acknowledgments}

This work is supported by the National Natural Science Foundation of China (no. 61471060) and Funds for Creative Research Groups of China (no. 61421061).

\section{References}

[1] C. Liang and F. R. Yu, "Wireless network virtualization: a survey, some research issues and challenges," IEEE Communications Surveys \& Tutorials, vol. 17, no. 1, pp. 358-380, 2015.

[2] C. Liang and F. R. Yu, "Wireless virtualization for next generation mobile cellular networks," IEEE Wireless Communications, vol. 22, no. 1, pp. 61-69, 2015.

[3] 3GPP, Technical Specification Group Services and System Aspects; Service aspects and requirements for network sharing, 3rd Generation Partnership Project (3GPP), TR 22.951 V11.0.0, September 2013, http://www.3gpp.org/ftp/specs/html-INFO/ 22951.htm.

[4] P. Rost, C. J. Bernardos, A. D. Domenico et al., "Cloud technologies for flexible $5 \mathrm{G}$ radio access networks," IEEE Communications Magazine, vol. 52, no. 5, pp. 68-76, 2014.

[5] D. Yun, J. Ok, B. Shin, S. Park, and Y. Yi, "Embedding of virtual network requests over static wireless multihop networks," Computer Networks, vol. 57, no. 5, pp. 1139-1152, 2013.

[6] G. Bhanage, D. Vete, I. Seskar, and D. Raychaudhuri, "Splitap: leveraging wireless network virtualization for flexible sharing of WLANs," in Proceedings of the IEEE Global Telecommunications Conference (GLOBECOM '10), pp. 1-6, Miami, Fla, USA, December 2010.

[7] V. Philip, Y. Gourhant, and D. Zeghlache, "OpenFlow as an architecture for e-node B virtualization," in e-Infrastructure and e-Services for Developing Countries, vol. 92 of Lecture Notes of the Institute for Computer Sciences, Social Informatics and Telecommunications Engineering, chapter 5, pp. 49-63, Springer, Berlin, Germany, 2012.
[8] R. Kokku, R. Mahindra, H. Zhang, and S. Rangarajan, "NVS: a substrate for virtualizing wireless resources in cellular networks," IEEE/ACM Transactions on Networking, vol. 20, no. 5, pp. 1333-1346, 2012.

[9] B. Liu and H. Tian, "A bankruptcy game-based resource allocation approach among virtual mobile operators," IEEE Communications Letters, vol. 17, no. 7, pp. 1420-1423, 2013.

[10] M. Kalil, A. Moubayed, A. Shami, and A. Al-Dweik, "Efficient low-complexity scheduler for wireless resource virtualization," IEEE Wireless Communications Letters, vol. 5, no. 1, pp. 56-59, 2016.

[11] L. Caeiro, F. D. Cardoso, and L. M. Correia, "Adaptive allocation of Virtual Radio Resources over heterogeneous wireless networks," in Proceedings of the 18th European Wireless Conference on European Wireless (EW '12), pp. 1-7, Poznan, Poland, April 2012.

[12] NGMN, "Suggestions on potential solutions to C-RAN by NGMN alliance," 2013, https://www.ngmn.org/uploads/media/ NGMN_CRAN_Suggestions_on_Potential_Solutions_to_CRAN .pdf.

[13] H. Yaïche, R. R. Mazumdar, and C. Rosenberg, "A game theoretic framework for bandwidth allocation and pricing in broadband networks," IEEE/ACM Transactions on Networking, vol. 8, no. 5, pp. 667-678, 2000.

[14] E. U. T. R. Access, "Further advance-ments for e-utra physical layer aspects,” Tech. Rep. 3GPP TR 36.814, 2010.

[15] D. Niyato and E. Hossain, "A noncooperative game-theoretic framework for radio resource management in $4 \mathrm{G}$ heterogeneous wireless access networks," IEEE Transactions on Mobile Computing, vol. 7, no. 3, pp. 332-345, 2008.

[16] J. W. K. Chan and T. K. L. Tong, "Multi-criteria material selections and end-of-life product strategy: grey relational analysis approach," Materials \& Design, vol. 28, no. 5, pp. 15391546, 2007.

[17] "IEEE standard for telecommunications and information exchange between systems-LAN/MAN specific requirements part 11: wireless medium access control (MAC) and physical layer (PHY) specifications: high speed physical layer in the 5 GHz band," IEEE Std 802.11a1999, 1999.

[18] R. Jain, W. Hawe, and D. Chiu, "A quantitative measure of fairness and discrimination of resource allocation in shared computer system," DEC Research Report TR-301, 1984. 

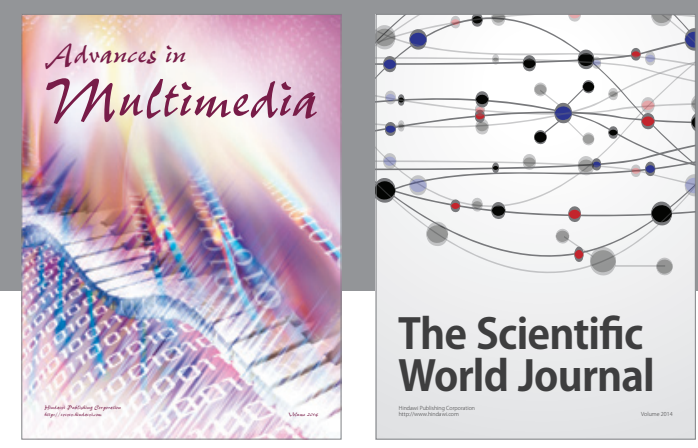

The Scientific World Journal
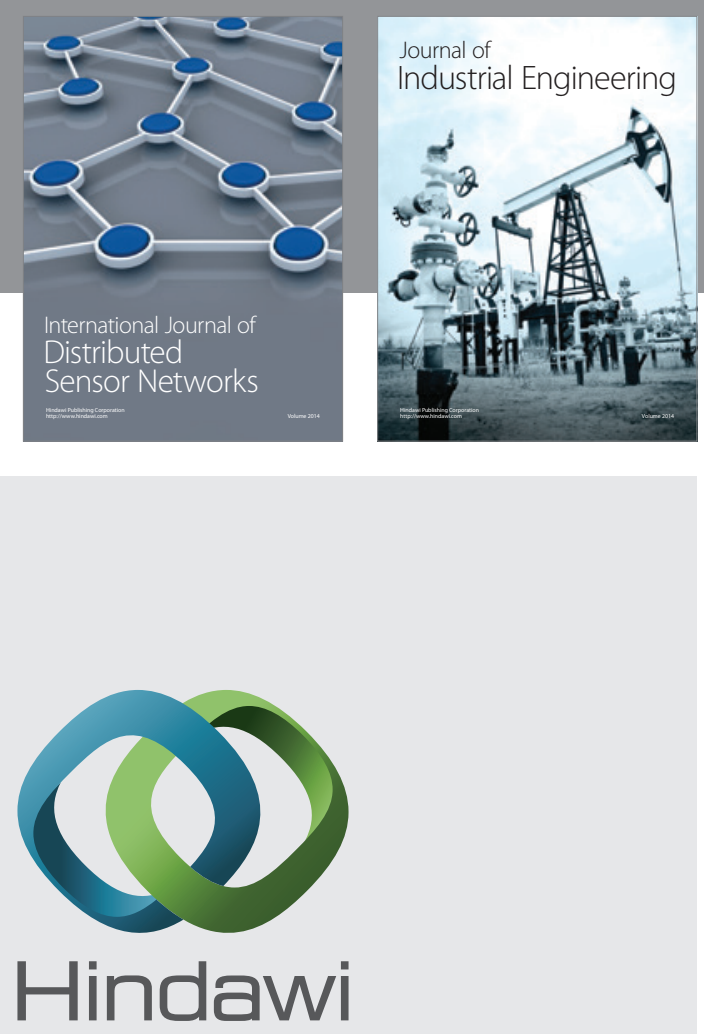

Submit your manuscripts at

http://www.hindawi.com

\section{Computer Networks} and Communications
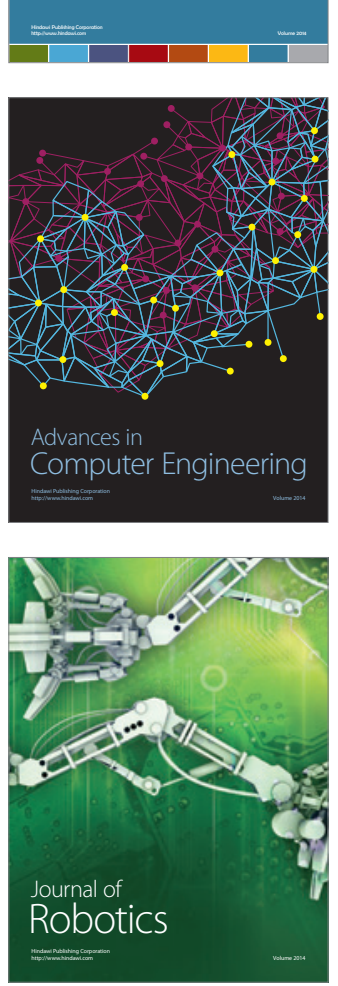
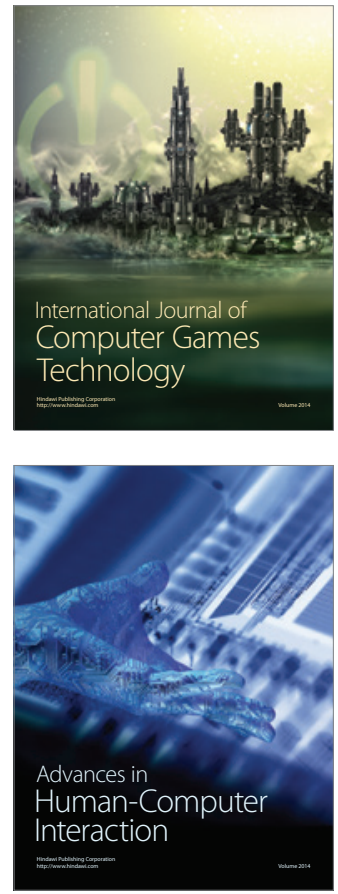
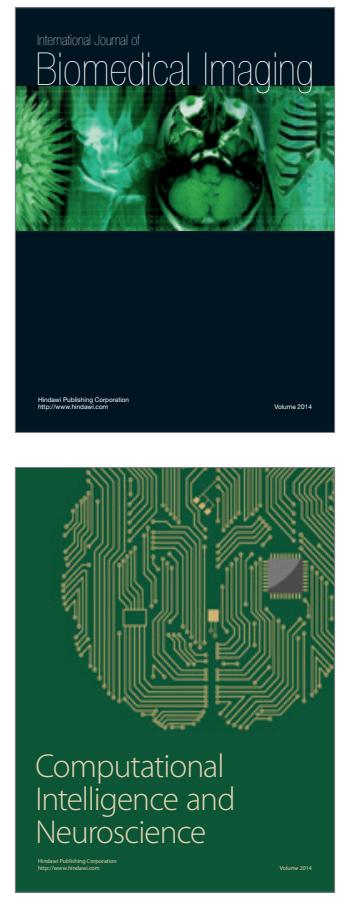
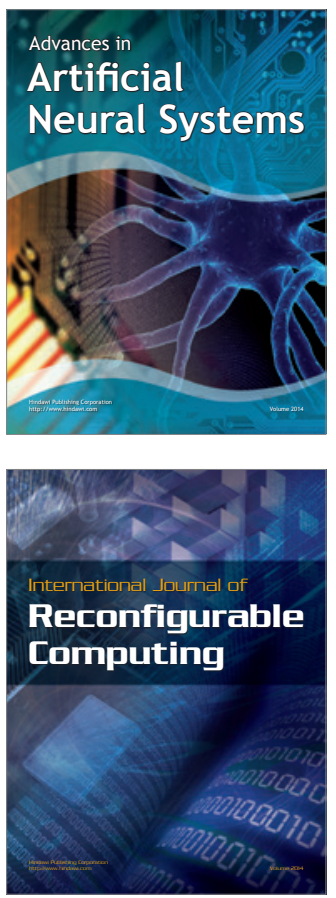
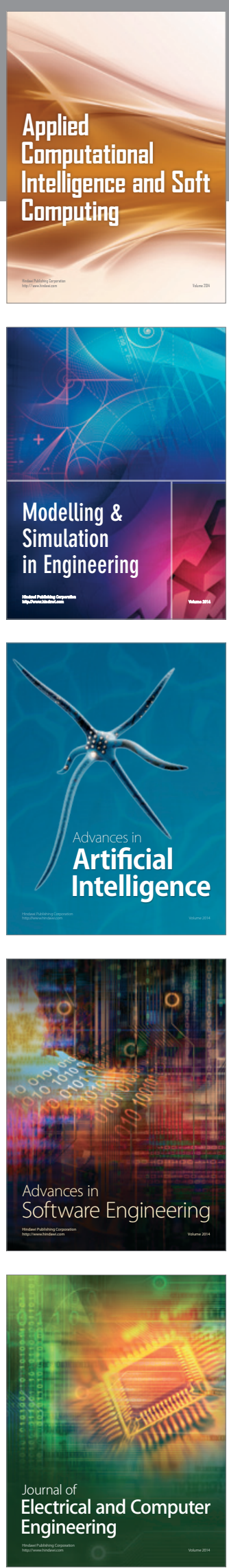